\title{
Frontal Dysfunction Underlies Depression in Mild Cognitive Impairment: A FDG-PET Study
}

\author{
Hye Sook Lee ${ }^{1}$, II Han Choo', Dong Young Lee ${ }^{1,2} \bowtie$, Jee Wook Kim¹, Eun Hyun Seo ${ }^{2}$, \\ Shin Gyeom Kim ${ }^{3}$, Shin Young Park ${ }^{4}$, Ji Hye Shin ${ }^{1}$, Ki Woong Kim ${ }^{5}$ and Jong Inn Woo ${ }^{1,2}$ \\ 'Departments of Neuropsychiatry and 'Interdisciplinary Program for Cognitive Science, College of Medicine, Seoul National University, Seoul, Korea \\ ${ }^{3}$ Department of Neuropsychiatry, College of Medicine, Soonchunhyang University, Bucheon Hospital, Bucheon, Korea \\ ${ }^{4}$ Department of Neuropsychiatry, Daerim St. Mary's Hospital, Seoul, Korea \\ ${ }^{5}$ Department of Neuropsychiatry, College of Medicine, Seoul National University, Bundang Hospital, Seongnam, Korea
}

Objective Depression is a very common symptom in people with mild cognitive impairment (MCI), a preclinical stage of Alzheimer's disease $(\mathrm{AD})$, and in those with clinically evident $\mathrm{AD}$. Moreover, $\mathrm{MCI}$ individuals with depression show a higher conversion rate to clinical AD than those without depression. This study aimed to elucidate the functional neuroanatomical substrate of depression in MCI.

Methods Thirty-six patients were recruited from a University Hospital-based cohort; 18 of these subjects had MCI with depression (MCI_D); the remaining 18 subjects were age- and gender-matched, and had MCI with no depression (MCI_ND). For comparison, 16 cognitively normal (CN) elderly individuals were also included. All subjects underwent Fluorodeoxyglucose Positron Emission Tomography (FDG-PET) scanning and regional cerebral glucose metabolism was compared among the three groups by a voxel-based method. The relationship between severity of depression, as measured by Hamilton Rating Scale for Depression (HRSD) scores, and glucose metabolism was also investigated.

Results MCI_D showed lower glucose metabolism in the right superior frontal gyrus than MCI_ND. There was a significant negative correlation between HRSD score and glucose metabolism at the same frontal region for overall MCI subjects. When compared with CN, both MCI_D and MCI_ND showed decreased glucose metabolism in the precuneus, while MCI_D had, in addition, reduced metabolism in other diffuse brain regions.

Conclusion Given previous observations on depression in $\mathrm{AD}$, our results suggest that functional disruption of the frontal region, known to be associated with primary or other secondary depression, underlies depression in preclinical AD as well as clinically evident AD.

Psychiatry Investig 2010;7:208-214

Key Words Mild cognitive impairment, Depression, Frontal, Fluorodeoxyglucose Positron Emission Tomography.

\section{INTRODUCTION}

Depression is a very common and significant psychiatric complication that affects 30 to $50 \%$ of Alzheimer's disease (AD) patients. ${ }^{1,2}$ Depression increases the suffering of $\mathrm{AD}$ patients and their families, and compounds consequent disability. While other psychiatric symptoms, such as delusion, hallucination, agitation, and apathy, occur mainly in the later

Received: February 1, 2010 Revised: April 26, 2010

Accepted: May 14, 2010 Available online: August 13, 2010

$\triangle$ Correspondence: Dong Young Lee, MD, PhD

Department of Neuropsychiatry, Seoul National University College of Medicine, 101 Daehak-ro, Jongno-gu, Seoul 110-744, Korea

Tel: +82-2-2072-2205, Fax: +82-2-744-7241, E-mail: selfpsy@snu.ac.kr

(a) This is an Open Access article distributed under the terms of the Creative Commons Attribution Non-Commercial License (http://creativecommons.org/licenses/bync/3.0) which permits unrestricted non-commercial use, distribution, and reproduction in any medium, provided the original work is properly cited. stages of $\mathrm{AD}$, depression is common even in the very early stage of the disease. Patients with mild cognitive impairment $(\mathrm{MCI})$, a preclinical stage of $\mathrm{AD}$, also frequently experience depression. The prevalence of depression in MCI has been reported to be $16-20 \% .{ }^{3,4} \mathrm{MCI}$ with depression has more than a two-fold higher $\mathrm{AD}$ conversion rate, compared to $\mathrm{MCI}$ with no depression. ${ }^{5,6}$

A couple of functional neuroimaging studies revealed that functional impairment of frontal cortical regions was associated with depression in $\mathrm{AD} .^{7-9} \mathrm{~A}$ previous report by our study group has indicated that depressive $\mathrm{AD}$ patients have lower glucose metabolism in the right superior frontal gyrus than non-depressive AD patients. ${ }^{7}$ Other $\left[{ }^{18} \mathrm{~F}\right]$ Fluorodeoxyglucose Positron Emission Tomography (FDG-PET) studies have also reported that superior frontal hypometabolism is associated with depression in $\mathrm{AD} .{ }^{89}$ Hirono et al. ${ }^{8}$ reported that de- 
pression was related to hypometabolism in bilateral superior frontal and left anterior cingulate cortices. Holthoff et al. ${ }^{9}$ reported that reduced glucose metabolism of dorsolateral prefrontal regions was associated with depression in $\mathrm{AD}$. However, little information is available that relates to the neural substrate of depression in MCI.

The aim of this study was to elucidate the functional neuroanatomical substrate of depression in MCI. We first compared regional cerebral glucose metabolism between $\mathrm{MCI}$ with depression (MCI_D) and MCI with no depression (MCI_ND) subjects, considering depression as a syndrome. We further investigated the linear relationship between depression severity and regional glucose metabolism in all MCI patients.

\section{METHODS}

\section{Subjects}

Thirty-six MCI patients including 18 MCI_D and 18 MCI ND cases matched for age and gender were recruited from a cohort regularly followed at the Dementia \& Age-Associated Cognitive Decline Clinic at Seoul National University Hospital. MCI was diagnosed according to current consensus criteria for amnestic MCI. ${ }^{10}$ Depression was diagnosed when a subject met the major or minor depressive disorder criteria of the fourth edition of the Diagnostic Statistical Manual of Mental Disorders 4th edition. All MCI individuals had an overall clinical dementia rating $(\mathrm{CDR})^{11}$ of 0.5 . Sixteen cognitively normal $(\mathrm{CN})$ elderly individuals were also recruited. All CN subjects received a CDR score of 0 and did not meet depression criteria.

The following exclusion criteria were applied to all subjects: any present serious medical or neurological disorder that could affect mental function; any major psychiatric disorders except depression, evidence of focal brain lesions on magnetic resonance imaging (MRI) including lacunes and white matter hyperintensity lesions of grade 2 or more on the Fazeka scale, ${ }^{12}$ the presence of severe behavioral or communication problems that would make a clinical or cerebral imaging of MRI and PET examination difficult; ambidextrousness or left-handedness; and the absence of a reliable informant. The Institutional Review Board of Seoul National University Hospital, approved the study protocol and informed consent was obtained from all study subjects and their relatives.

\section{Clinical and neuropsychological assessments}

All subjects received a standardized clinical assessment and MRI according to the protocol of the Korean version of the Consortium to Establish a Registry for Alzheimer's Disease (CERAD) Assessment Packet. ${ }^{13}$

The CERAD neuropsychological battery includes eight neu- ropsychological tests: Verbal Fluency; 15-item Boston Naming Test; Mini-Mental State Examination (MMSE); World List Memory; Word List Recall; Word List Recognition; Constructional Praxis (CP); Constructional Recall, and was administered by experienced clinical psychologists. Depression severity was assessed using the 17-item Hamilton Rating Scale for Depression (HRSD) $)^{14}$ included in Clinical Assessment of Depression in Dementia. ${ }^{2}$ A panel consisting of four neuropsychiatrists with expertise in dementia research made clinical decisions, including the assignment of clinical diagnosis and CDR rating. All clinical assessments were carried out within 4 weeks of PET examination.

\section{PET imaging}

PET studies were performed using an ECAT EXACT 47 scanner (Siemens-CTI, Knoxville, TN) with an intrinsic resolution of a $5.2 \mathrm{~mm}$ full width at half maximum and 47 contiguous transverse plane images with a $3.4 \mathrm{~mm}$ thickness for a longitudinal field of view of $16.2 \mathrm{~cm}$. Before administering FDG, transmission scanning was performed, using 3 germanium-68 rod sources to correct attenuation. Static emission scans began 30 minutes after the intravenous injection of 370 MBq (10 mCi) FDG and were continued for 30 minutes. All FDG-PET scans were performed in a dimly lit room with minimal auditory stimulation during PET scanning, with the subject in the supine position with their eyes closed to minimize confounding effects due to activity. Transaxial images were reconstructed using a filtered back-projection algorithm employing a Shepp-Logan filter with a cutoff frequency of 0.3 cycles/ pixel and $128 \times 128 \times 47$ matrices of size of $2.1 \times 2.1 \times 3.4 \mathrm{~mm}$.

\section{PET data analysis}

Imaging data were analyzed using Statistical Parametric Mapping (SPM) 2 (Institute of Neurology, University College of London, UK) implemented in the Matlab (Mathworks Inc, USA). Before statistical analysis, all images were spatially normalized to the Montreal Neurological Institute (MNI, McGill University, Montreal, CA, USA) space to correct intersubject anatomical variabilities. An affine transformation was performed to determine the 12 optimal parameters essential for registering the brain on the MNI template. Subtle differences between transformed images and the template were removed by a nonlinear registration method using the weighted sum of predefined smooth basis functions used in a discrete cosine transformation. Using an in-house Matlab-based program, the glucose metabolism value of each voxel was normalized for pontine value, which was extracted for each scan, because glucose metabolism in the pons tends to be relatively preserved in $\mathrm{AD} .{ }^{15}$ In this program, the volume of interest for the pons was predefined in the MNI space and applied to the in- 
dividual spatially normalized PET images to measure the mean pontine activity. Normalized images were smoothed by convolution using an isotropic Gaussian kernel with 16 $\mathrm{mm}$ full width at half maximum to accommodate intersubject differences in gyral and functional anatomies and to increase dataset signal-to noise ratios.

Differences in glucose metabolic values between MCI_D, MCI_ND and CN were estimated on a voxel-by-voxel basis using the t-test. The resulting set of $t$ values constituted the SPM(t) map. The SPM(t) was then transformed into a normal distribution to give a $\operatorname{SPM}(\mathrm{Z}) .{ }^{16}$ We applied both $\mathrm{p}$ value $<0.001$ (uncorrected for multiple comparisons) as a significance height threshold and 50 voxels as a cluster size threshold to decrease the probability of detecting false positives. ${ }^{17,18}$ The MNI coordinates of the local maximum of each voxel cluster were automatically calculated in SPM, and could be transformed to Talairach and Taurnoux ${ }^{19}$ by "min2tal" program (http://imaging. mrc-cbu.cam.ac.uk/downloads/MNI2tal/).

To further characterize regional glucose metabolic alterations associated with depression, we extracted normalized metabolism values at the local maximum of voxel cluster showing a significant difference between MCI_D and MCI_ND in voxel-by-voxel analysis using the voxel of interest module of SPM. The extracted metabolism values of the region were compared between MCI_D, MCI_ND and CN using analysis of variance (ANOVA) and post hoc contrasts with Tukey's method using Statistical Package for the Social Sciences (SPSS, SPSS Inc., Chicago, IL, USA). A partial correlation between depression severity, as measured by HRSD, and the extracted metabolism values was also assessed, controlling for age as a covariate for all the MCI subjects, using SPSS. In these analyses of extracted values, a $p$ value of $<0.05$ was applied as the threshold of statistical significance.

\section{Other data analysis}

The demographic and clinical data from MCI_D, MCI_ ND and CN subjects were compared by ANOVA and post hoc contrasts with Tukey's method for continuous variables and $\chi^{2}$ tests for categorical variables using SPSS.

\section{RESULTS}

\section{Demographic and clinical characteristics of the subjects}

Demographic and clinical characteristics for subjects are presented in Table 1. MCI_D, MCI_ND and CN did not differ in regard to age, gender or education. For all eight neuropsychological tests, including MMSE, there was no significant difference between the MCI_D and MCI_ND groups. In contrast, both MCI_D and MCI_ND had significantly lower scores than $\mathrm{CN}$ for most of the neuropsychological tests except CP. Only MCI_D showed lower CP score than CN, but MCI ND did not. MCI_D subjects also had significantly higher HRSD scores than did either MCI_ND or CN individuals.

\section{Cerebral glucose metabolic changes associated with depression in MCI}

Voxel-wise comparison revealed that MCI_D had signifi-

Table 1. Demographic and clinical characteristics of subjects

\begin{tabular}{|c|c|c|c|c|}
\hline & MCI_D & MCI_ND & $\mathrm{CN}$ & $\mathrm{p}$ value \\
\hline No. $(F / M)$ & $18(14 / 4)$ & $18(12 / 6)$ & $16(9 / 7)$ & NS \\
\hline Age (y) & $69.4 \pm 9.5$ & $69.4 \pm 10.1$ & $72.3 \pm 5.2$ & NS \\
\hline Education (y) & $9.7 \pm 4.0$ & $8.6 \pm 5.0$ & $11.5 \pm 4.1$ & NS \\
\hline HRSD score & $12 \pm 4.0$ & $2.6 \pm 3.0$ & $0.63 \pm 1.5$ & $<0.05^{*+}$ \\
\hline \multicolumn{5}{|l|}{ Neuropsychiatric tests } \\
\hline Word fluency & $39.9 \pm 11.2$ & $44.2 \pm 7.8$ & $58.4 \pm 11.1$ & $<0.05^{\dagger \ddagger}$ \\
\hline 15-item Boston Naming & $44.2 \pm 12.5$ & $46.9 \pm 9.9$ & $57.6 \pm 6.7$ & $<0.05^{\dagger \ddagger}$ \\
\hline Mini-Mental State & $22.7 \pm 22.3$ & $33.1 \pm 14.5$ & $52.3 \pm 7.5$ & $<0.05^{\dagger \ddagger}$ \\
\hline Word List Memory & $40.6 \pm 13.4$ & $40.6 \pm 10.2$ & $61.4 \pm 8.9$ & $<0.05^{\text {} \ddagger}$ \\
\hline Constructional Praxis & $46.3 \pm 13.2$ & $49.3 \pm 10.0$ & $55.6 \pm 4.3$ & $<0.05^{\dagger}$ \\
\hline Word List Recall & $41.3 \pm 10.2$ & $35.3 \pm 10.2$ & $58.0 \pm 6.2$ & $<0.05^{\dagger \ddagger}$ \\
\hline Word List Recognition & $40.5 \pm 14.6$ & $35.6 \pm 16.6$ & $52.8 \pm 7.1$ & $<0.05^{\text {} \ddagger}$ \\
\hline Constructional Recall & $36.3 \pm 9.6$ & $41.4 \pm 8.9$ & $54.0 \pm 7.2$ & $<0.05^{\dagger \ddagger}$ \\
\hline
\end{tabular}

Data are presented as mean \pm SD. All neuropsychological test scores are age, education and gender-specific norm corrected t-tests. Group comparisons are by analysis of variance. Post hoc comparisons of significant group differences. * MCI_D vs. MCI_ND, ${ }^{\dagger} \mathrm{MCI} D \mathrm{D}$ vs. CN, ${ }^{\ddagger}$ MCI_ND vs. CN. HRSD: Hamilton Rating Scale for Depression, MCI_D: mild cognitive impairment with depression, MCI_ND: mild cognitive impairment with no depression, $\mathrm{CN}$ : cognitively normal 
Table 2. Brain areas showing significant hypometabolism in group comparisons

\begin{tabular}{|c|c|c|c|c|c|c|c|}
\hline \multirow{2}{*}{ Brain region } & \multirow{2}{*}{$\mathrm{BA}$} & \multicolumn{3}{|c|}{ Coordinates (mm) } & \multirow{2}{*}{ Voxels (N) } & \multirow{2}{*}{ Z score } & \multirow{2}{*}{$\begin{array}{c}\mathrm{p} \text { value } \\
\text { (uncorrected }\end{array}$} \\
\hline & & $\mathrm{X}$ & $\mathrm{Y}$ & $\mathrm{Z}$ & & & \\
\hline \multicolumn{8}{|l|}{ MCI_D vs. MCI_ND } \\
\hline Right superior frontal gyrus & 6 & 30.0 & -8.0 & -69.0 & 29 & 3.35 & $<0.001$ \\
\hline \multicolumn{8}{|l|}{ MCI_D vs. CN } \\
\hline Right precuneus & 31 & 14.0 & -53.0 & 32.0 & 1,198 & 4.02 & $<0.001$ \\
\hline Left precuneus & 7 & -24.0 & -68.0 & 35.0 & 1,108 & 3.65 & $<0.001$ \\
\hline Right superior parietal gyrus & 6 & 65.0 & 3.0 & 15.0 & 645 & 3.49 & $<0.001$ \\
\hline Left postcentral gyrus & 47 & 50.0 & 48.0 & -10.0 & 679 & 3.43 & $<0.001$ \\
\hline Left fusiform gyrus & 39 & 50.0 & -61.0 & 18.0 & 516 & 3.38 & $<0.001$ \\
\hline Left parahippocampal gyrus & 6 & 26.0 & -14.0 & 69.0 & 126 & 3.32 & $<0.001$ \\
\hline \multicolumn{8}{|l|}{ MCI_ND vs.CN } \\
\hline Right precuneus & 31 & 16.0 & -57.0 & 34.0 & 29 & 3.18 & 0.001 \\
\hline
\end{tabular}

Coordinates (x,y and $\mathrm{z}$ ) refer to a standard stereotactical space. ${ }^{19}$ Each coordinate indicate the voxel location with the highest $\mathrm{z}$ score within each brain region. MCI_D: mild cognitive impairment with depression, MCI_ND: mild cognitive impairment with no depression, CN: cognitively normal

Figure 1. Statistical parametric maps showing decreased glucose metabolism in mild cognitive impairment $(\mathrm{MCl})$ with depression compared with $\mathrm{MCl}$ without depression at $p<0.001$ (uncorrected).
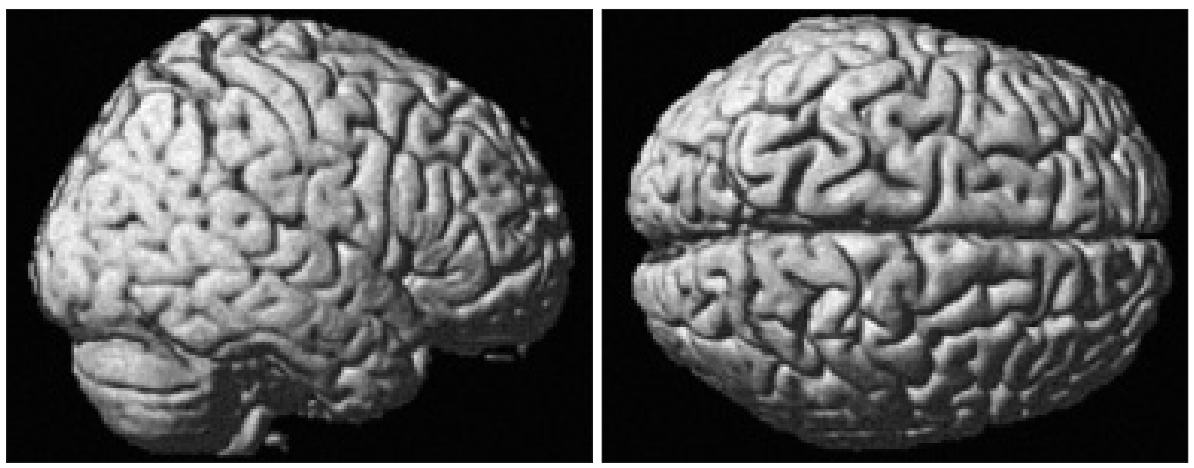

cantly lower glucose metabolism in the right superior frontal region [Brodmann's area (BA) 6] than MCI_ND (Table 2, Figure 1). In contrast, there was no brain region where MCI_D showed increased glucose metabolism compared with MCI_ ND. Voxel-based analysis also showed that MCI_D had significantly lower glucose metabolism in the bilateral precuneus (BA 31 and BA 7), right superior parietal gyrus (BA 6), left postcentral gyrus (BA 47), left fusiform gyrus (BA 39), and left parahippocampal gyrus (BA 6) than CN. MCI_ND also showed marginally significant hypometabolism in the right precuneus (BA 31), compared with CN (Table 2).

To characterize further glucose metabolic alteration associated with depression on the right superior frontal region, we extracted normalized metabolism values at the local maximum of voxel cluster showing a significant difference between MCI_D and MCI_ND groups using the voxel of interest module of SPM. There was a significant between-group difference in the extracted regional metabolism value between $\mathrm{MCI}_{-}$ D, MCI_ND, and CN groups $(\mathrm{F}=7.87, \mathrm{df}=2 ; \mathrm{p}<0.005)$. As shown in Figure 2, both MCI_ND and CN groups had signifi-

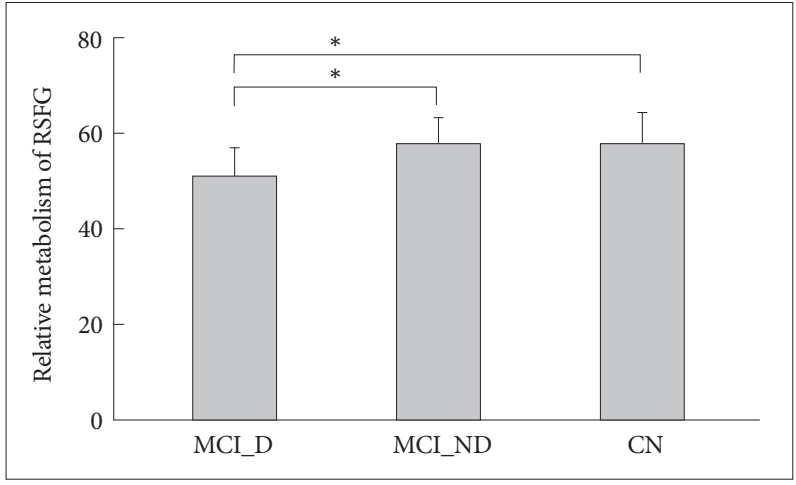

Figure 2. Group comparisons of glucose metabolism in the right superior frontal region. Error bars indicate SDs. ${ }^{*} p<0.005$ by Tukey's post hoc group comparison. RSFG: right superior frontal gyrus, $\mathrm{MCl}$ D: mild cognitive impairment with depression, MCl_ND: mild cognitive impairment with no depression, $\mathrm{CN}$ : cognitively normal.

cantly higher glucose metabolism than MCI_D, while there was no significant difference between MCI_ND and CN groups. There was also a significant correlation between HRSD scores and the extracted metabolism values in overall MCI subjects ( artial correlation coefficient $=-0.418 ; \mathrm{p}=0.012$ )(Figure 3 ). 


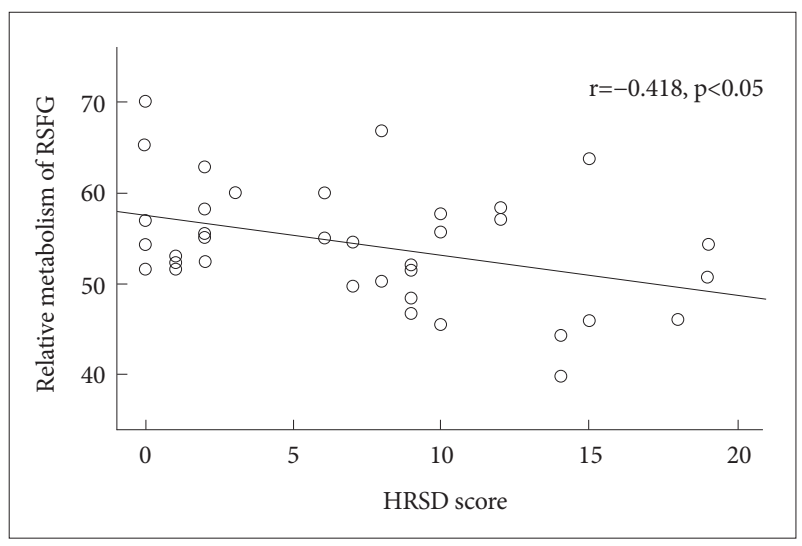

Figure 3. Illustration of the significant correlation obtained between depression severity (Hamilton Rating Scale for Depression scores) and glucose metabolism in the right superior frontal gyrus in mild cognitive impairment subjects overall. HRSD: Hamilton Rating Scale for Depression.

\section{DISCUSSION}

Through a voxel-based approach, we found that MCI_D had significantly lower glucose metabolism in the right superior frontal region than MCI_ND. Furthermore, depression severity as measured by HRSD showed a significant negative correlation with glucose metabolism in the same brain region. To the best of our knowledge, this is the first study that reveals the functional neuro-anatomical substrate associated with depression in MCI.

The finding that reduced frontal glucose metabolism is related to depression in MCI is generally in line with the results of previous neuro-imaging studies on secondary depression, as well as primary depression, although the exact locations relating to depression within the frontal cortex vary among the studies. ${ }^{7-9,20-24}$ More specifically, focusing on $\mathrm{AD}$ process-related depression, a previous FDG-PET study by our group ${ }^{7}$ indicated that depression in $\mathrm{AD}$ is associated with hypometabolism in the right superior frontal region (BA 6), the very region related to depressive $\mathrm{MCI}$ in the current study. Other FDG-PET studies ${ }^{8,9}$ also demonstrated that depression score correlates significantly with glucose hypometabolism in the bilateral superior frontal or dorsolateral prefrontal cortices. In one single photon emission computerized tomography study, depressed $\mathrm{AD}$ patients showed lower perfusion in the bilateral superior frontal gyrus than non-depressed $\mathrm{AD}$ patients. ${ }^{24}$ These converging results from both our $\mathrm{MCI}$ and previous $\mathrm{AD}$ studies on depression correspond well with the fact that amnestic $\mathrm{MCI}$ is a preclinical or high risk state of $\mathrm{AD}{ }^{25-27}$

Although BA 6 has long been recognized as a premotor or high-order motor area, this does not necessarily indicate that motor system deficit has a close relationship with depression in MCI or early $\mathrm{AD}$ process. ${ }^{7}$ The rostral parts of $\mathrm{BA} 6$, where depression-related hypometabolism was observed in the cur- rent study, are closely interconnected with the prefrontal cortex rather than the primary motor cortex, while the caudal parts of BA 6 have a close relationship with the primary motor cortex. ${ }^{7.28}$ Therefore, it seems more likely that the deficit of rostral BA 6 in depressive $\mathrm{MCI}$ is related to prefrontal dysfunction than to motor system dysfunction. A recent report showed that loss of motivated behavior including work, activities, appetite and libido in primary depression correlated with decreased glucose metabolism in BA $6{ }^{29}$

While MCI_D showed superior frontal hypometabolism compared with MCI_ND, voxel-based analysis did not reveal any metabolic differences in the same region between MCI $\mathrm{D}$ and $\mathrm{CN}$ or between MCI_ND and CN. The lack of a significant finding in this regard is probably related to the threshold for statistical significance used in voxel-based analysis. Our study applied a relatively strict threshold $(\mathrm{p}<0.001)$ to reduce the possibility of false positive results (alpha error) due to multiple comparisons. However, this increases the possibility of false negative results (beta error). When we re-analyzed with a less strict statistical threshold ( $\mathrm{p}<0.005$, by ANOVA with Tukey's post hoc comparison) focusing on the right superior frontal region, MCI_D patients showed significantly lower metabolism than either CN or MCI_ND individuals.

Both MCI_D and MCI_ND showed lower glucose metabolism in the precuneus than $\mathrm{CN}$. This finding corresponds with the results of earlier studies, which reported glucose hypometabolism in the medial parietal region including the precuneus and posterior cingulate cortex in MCI and very early AD. ${ }^{30-34}$ It also lends credence to the assumption that the MCI subjects recruited in our study represent preclinical AD patients. In addition, compared with CN, MCI_D patients showed hypometabolism in parieto-temporal regions other than the precuneus, but MCI_ND patients did not. This finding, together with depression-associated frontal hypometabolism in MCI detected through direct comparison, might suggest that MCI_D have a more diffuse deposition of AD related pathologies than MCI_ND, although there were no significant differences in apparent neuropsychological test performances between MCI_D and MCI_ND (Table 1). Compatible with this, previous studies reported that parieto-temporal hypometabolism found in MCI patients was associated with increased progression to clinical $\mathrm{AD},{ }^{35-37}$ and that the presence of depression in MCI patients increased the risk for conversion to clinical AD. ${ }^{5,6}$ Also, there were a couple of reports that amyloid beta deposition, a major component of neuritic plaque, was associated with late-life depression..$^{38,39}$

There are some possible limitations to the present study. First, the relatively small sample size and potential selection bias related to subject recruitment from a tertiary hospital cohort mean caution is required about generalizing from the re- 
sults. In particular, low statistical power due to small sample size may make it difficult to detect other regional brain substrates less strongly associated with depression in MCI than the right superior frontal region. Second, we did not apply partial volume effect (PVE) correction to the PET data and, therefore, brain hypometabolism in MCI might be overestimated, compared with $\mathrm{CN}$. However, given that overall brain atrophy is less prominent in MCI, and that PVE due to brain atrophy does not alter the general pattern of differences of metabolism in $\mathrm{AD}$, especially in mild cases, ${ }^{40}$ it is not likely to affect our results to any great degree.

In conclusion, our finding of frontal hypometabolism associated with depression in MCI corresponds with previous reports on depression in $\mathrm{AD}$ and suggests that the functional disruption of the frontal region is associated with depression in preclinical $\mathrm{AD}$, as well as in clinically evident $\mathrm{AD}$. When combined with previous observations on primary and secondary depression, these results suggest that frontal dysfunction is probably associated with depression, regardless of the etiology.

\section{Acknowledgments}

This study was supported grants from the Korea Healthcare Technology R \& D Project, Ministry of Health, Welfare \& Family Affairs, Republic of Korea (Grant No. A070001 \& A092145).

\section{REFERENCES}

1. Olin JT, Schneider LS, Katz IR, Meyers BS, Alexopoulos GS, Breitner JC, et al. Provisional diagnostic criteria for depression of Alzheimer disease. Am J Geriatr Psychiatry 2002;10:125-128.

2. Zubenko GS, Zubenko WN, McPherson S, Spoor E, Marin DB, Farlow MR, et al. A collaborative study of the emergence and clinical features of the major depressive syndrome of Alzheimer's disease. Am J Psychiatry 2003;160:857-866.

3. Chan DC, Kasper JD, Black BS, Rabins PV. Presence of behavioral and psychological symptoms predicts nursing home placement in community-dwelling elders with cognitive impairment in univariate but not multivariate analysis. J Gerontol A Biol Sci Med Sci 2003;58:548-554.

4. Lyketsos CG, Lopez O, Jones B, Fitzpatrick AL, Breitner J, DeKosky S. Prevalence of neuropsychiatric symptoms in dementia and mild cognitive impairment: results from the cardiovascular health study. JAMA 2002;288:1475-1483.

5. Modrego PJ, Ferrández J. Depression in patients with mild cognitive impairment increases the risk of developing dementia of Alzheimer type: a prospective cohort study. Arch Neurol 2004;61:1290-1293.

6. Gabryelewicz T, Styczynska M, Luczywek E, Barczak A, Pfeffer A, Androsiuk W, et al. The rate of conversion of mild cognitive impairment to dementia: predictive role of depression. Int J Geriatr Psychiatry 2007;22:563-567.

7. Lee DY, Choo IH, Jhoo JH, Kim KW, Youn JC, Lee DS, et al. Frontal dysfunction underlies depressive syndrome in Alzheimer disease: a FDG-PET study. Am J Geriatr Psychiatry 2006;14:625-628.

8. Hirono N, Mori E, Ishii K, Ikejiri Y, Imamura T, Shimomura T, et al. Frontal lobe hypometabolism and depression in Alzheimer's disease. Neurology 1998;50:380-383.

9. Holthoff VA, Beuthien-Baumann B, Kalbe E, Lüdecke S, Lenz O, Zündorf G, et al. Regional cerebral metabolism in early Alzheimer's disease with clinically significant apathy or depression. Biol Psychiatry 2005; 57:412-421.
10. Winblad B, Palmer K, Kivipelto M, Jelic V, Fratiglioni L, Wahlund LO, et al. Mild cognitive impairment--beyond controversies, towards a consensus: report of the International Working Group on Mild Cognitive Impairment. J Intern Med 2004;256:240-246.

11. Morris JC. The Clinical Dementia Rating (CDR): current version and scoring rules. Neurology 1993;43:2412-2414.

12. Fazekas F, Chawluk JB, Alavi A, Hurtig HI, Zimmerman RA. MR signal abnormalities at $1.5 \mathrm{~T}$ in Alzheimer's dementia and normal aging. AJR Am J Roentgenol 1987;149:351-356.

13. Lee JH, Lee KU, Lee DY, Kim KW, Jhoo JH, Kim JH, et al. Development of the Korean version of the Consortium to Establish a Registry for Alzheimer's Disease Assessment Packet (CERAD-K): clinical and neuropsychological assessment batteries. J Gerontol B Psychol Sci Soc Sci 2002;57:p47-p53.

14. Hamilton M. Development of a rating scale for primary depressive illness. Br J Soc Clin Psychol 1967;6:278-296.

15. Minoshima S, Frey KA, Foster NL, Kuhl DE. Preserved pontine glucose metabolism in Alzheimer disease: a reference region for functional brain image (PET) analysis. J Comput Assist Tomogr 1995;19:541-547.

16. Friston KJ, Holmes AP, Worsley KJ, Poline JP, Firth CD, Frackowiak RSJ. Statistical parametric maps in functional imaging: a general linear approach. J Hum Brain Mapp 1995;2:189-210.

17. Friston KJ, Frith CD, Liddle PF, Frackowiak RS. Comparing functional (PET) images: the assessment of significant change. J Cereb Blood Flow Metab 1991;11:690-699.

18. Forman SD, Cohen JD, Fitzgerald M, Eddy WF, Mintun MA, Noll DC. Improved assessment of significant activation in functional magnetic resonance imaging (fMRI): use of a cluster-size threshold. Magn Reson Med 1995;33:636-647.

19. Talairach J, Tournoux P. Co-planar Stereotaxic Atlas of the Human brain. New York: Theime; 1988.

20. Beblo T, Wallesch CW, Herrmann M. The crucial role of frontostriatal circuits for depressive disorders in the postacute stage after stroke. Neuropsychiatry Neuropsychol Behav Neurol 1999;12:236-246.

21. Mayberg HS, Starkstein SE, Sadzot B, Preziosi T, Andrezejewski PL, Dannals RF, et al. Selective hypometabolism in the inferior frontal lobe in depressed patients with Parkinson's disease. Ann Neurol 1990;28:5764.

22. Ring HA, Bench CJ, Trimble MR, Brooks DJ, Frackowiak RS, Dolan RJ. Depression in Parkinson's disease. A positron emission study. Br J Psychiatry 1994;165:333-339.

23. Fitzgerald PB, Oxley TJ, Laird AR, Kulkarni J, Egan GF, Daskalakis ZJ. An analysis of functional neuroimaging studies of dorsolateral prefrontal cortical activity in depression. Psychiatry Res 2006;148:33-45.

24. Levy-Cooperman N, Burhan AM, Rafi-Tari S, Kusano M, Ramirez J, Caldwell C, et al. Frontal lobe hypoperfusion and depressive symptoms in Alzheimer disease. J Psychiatry Neurosci 2008;33:218-226.

25. Gauthier S, Reisberg B, Zaudig M, Petersen RC, Ritchie K, Broich K, et al. Mild cognitive impairment. Lancet 2006;367:1262-1270.

26. Petersen RC. Mild cognitive impairment as a diagnostic entity. J Intern Med 2004;256:183-194.

27. Davis HS, Rockwood K. Conceptualization of mild cognitive impairment: a review. Int J Geriatr Psychiatry 2004;19:313-319.

28. Lu MT, Preston JB, Strick PL. Interconnections between the prefrontal cortex and the premotor areas in the frontal lobe. J Comp Neurol 1994; 341:375-392.

29. Milak MS, Parsey RV, Keilp J, Oquendo MA, Malone KM, Mann JJ. Neuroanatomic correlates of psychopathologic components of major depressive disorder. Arch Gen Psychiatry 2005;62:397-408.

30. Minoshima S, Giordani B, Berent S, Frey KA, Foster NL, Kuhl DE. Metabolic reduction in the posterior cingulate cortex in very early Alzheimer's disease. Ann Neurol 1997;42:85-94.

31. Chetelat G, Desgranges B, de la Sayette V, Viader F, Berkouk K, Landeau $B$, et al. Dissociating atrophy and hypometabolism impact on episodic memory in mild cognitive impairment. Brain 2003;126:1955- 
1967.

32. Ishii K, Mori T, Hirono N, Mori E. Glucose metabolic dysfunction in subjects with a clinical dementia rating of 0.5 . J Neurol Sci 2003;215: 71-74.

33. Nestor PJ, Fryer TD, Ikeda M, Hodges JR. Retrosplenial cortex (BA 29/30) hypometabolism in mild cognitive impairment (prodromal Alzheimer's disease). Eur J Neurosci 2003;18:2663-2667.

34. Herholz K, Carter SF, Jones M. Positron emission tomography imaging in dementia. Br J Radiol 2007;80:S160-S167.

35. Chételat G, Desgranges B, de la Sayette V, Viader F, Eustache F, Baron JC. Mild cognitive impairment: can FDG-PET predict who is to rapidly convert to Alzheimer's disease? Neurology 2003;60:1374-1377.

36. Arnáiz E, Jelic V, Almkvist O, Wahlund LO, Winblad B, Valind S, et al. Impaired cerebral glucose metabolism and cognitive functioning predict deterioration in mild cognitive impairment. Neuroreport 2001;12:
851-855.

37. Anchisi D, Borroni B, Franceschi M, Kerrouche N, Kalbe E, BeuthienBeumann B, et al. Heterogeneity of brain glucose metabolism in mild cognitive impairment and clinical progression to Alzheimer disease. Arch Neurol 2005;62:1728-1733.

38. Sweet RA, Hamilton RL, Butters MA, Mulsant BH, Pollock BG, Lewis $\mathrm{DA}$, et al. Neuropathologic correlates of late-onset major depression. Neuropsychopharmacology 2004;29:2242-2250.

39. Butters MA, Klunk WE, Mathis CA, Price JC, Ziolko SK, Hoge JA, et al. Imaging Alzheimer pathology in late-life depression with PET and Pittsburgh Compound-B. Alzheimer Dis Assoc Disord 2008;22:261-268.

40. Ibáñez V, Pietrini P, Alexander GE, Furey ML, Teichberg D, Rajapakse JC, et al. Regional glucose metabolic abnormalities are not the result of atrophy in Alzheimer's disease. Neurology 1998;50:1585-1593. 